\title{
Magnetic moments and shape coexistence in the light $\mathrm{Br}$ isotopes
}

\author{
Austyn G. Griffiths, Christopher J. Ashworth, J. Rikovska, N. J. Stone, and J. P. White \\ Clarendon Laboratory, Oxford University, Oxford OX1 3PU, United Kingdom \\ I. S. Grant \\ Schuster Laboratory, University of Manchester, Manchester M13 9PL, United Kingdom \\ P. M. Walker \\ Physics Department, University of Surrey, Guildford, Surrey GU2 5XH, United Kingdom \\ W. B. Walters \\ Chemistry Department, University of Maryland, College Park, Maryland 20742
}

(Received 1 May 1992)

\begin{abstract}
Low temperature nuclear orientation measurements have been made to study the nuclear magnetic dipole moments of ${ }^{72} \mathrm{Br}^{8, m},{ }^{73,74} \mathrm{Br}^{m},{ }^{75,76} \mathrm{Br}^{8}$, and ${ }^{77} \mathrm{Br}^{8}$ implanted into $\mathrm{Fe}$. The analysis of the data has been performed within the framework of a two, nonzero field, site model to describe the occupation of $\mathrm{Br}$ nuclei in the host $\mathrm{Fe}$ lattice. The two sites were associated with magnetic hyperfine fields of $\pm 81.38(6)$ and $\pm 26(2) \mathrm{T}$. A spin of $\frac{1}{2}$ for the ${ }^{73} \mathrm{Br}$ ground state is deduced and magnetic dipole moments are determined for the remaining nuclides. In addition, conversion electrons were observed for ${ }^{72} \mathrm{Br}$, confirming the multipolarity of the isomeric transition. An interpretation of the ground-state configurations and shapes of these isotopes is given using particle-rotor calculations and it is shown that the $\mathrm{Br}$ nuclides can be better described as a hole in the more deformed $\mathrm{Kr}$ nuclides rather than a particle beyond the Se cores.
\end{abstract}

PACS number(s): 21.10.Ky, 21.10.Hw, 23.20.En, 27.50. $+\mathrm{e}$

\section{INTRODUCTION}

Among the nuclei off the line of stability, those in the $Z \sim N \sim 40$ region are of particular interest, exhibiting large quadrupole deformations, possible triaxiality, and shape coexistence [1-5]. The nuclear ground-state spins of the odd- $A$ bromine nuclides ${ }^{75-87} \mathrm{Br}$ with $Z=35$ are all $\frac{3}{2}^{-}$. Nilsson orbital calculations show that the last odd proton occupies the [301] $\frac{3}{2}$ orbital for deformations $\varepsilon<0.22$ and the [312] $\frac{3}{2}$ orbital at larger prolate deformations. The magnetic dipole moments of these two configurations differ by a factor of 3 and thus clearly identify the single-particle configuration and hence the approximate deformation of the ground state.

With this aim static low temperature nuclear orientation (LTNO) measurements have been performed on the light bromine isotopes ${ }^{72} \mathrm{Br}^{g, m},{ }^{73,74} \mathrm{Br}^{m},{ }^{75,76} \mathrm{Br}^{g}$, and ${ }^{77} \mathrm{Br}^{g}$. These data are analyzed in terms of the best currently available models and supersede all preliminary results quoted in earlier reports [6,7]. A detailed theoretical interpretation of the data is also discussed.

\section{EXPERIMENTAL DETAILS}

Sources of ${ }^{76} \mathrm{Br}^{g}\left(t_{1 / 2}=16 \mathrm{~h}\right)$ and ${ }^{77} \mathrm{Br}^{g}\left(t_{1 / 2}=57 \mathrm{~h}\right)$, produced in the reaction of $600-\mathrm{MeV}$ protons on a target of niobium powder, were implanted at room temperature into polycrystalline iron foils at the ISOLDE facility, CERN. These were subsequently studied in Oxford at temperatures down to $2 \mathrm{mK}$ using a ${ }^{3} \mathrm{He}-{ }^{4} \mathrm{He}$ dilution refrigerator in conjunction with a $\mathrm{PrNi}_{5}$ demagnetization stage [8]. Data were collected from two $\mathrm{Ge}(\mathrm{Li})$ detectors positioned axially and equatorially relative to the axis of orientation, defined by a 1.0-T polarizing field. The source to detector distance was $12 \mathrm{~cm}$.

The isotopes ${ }^{72} \mathrm{Br}^{g, m}\left(t_{1 / 2}=1.3 \mathrm{~min}, 10.1 \mathrm{~s}\right.$, respective$1 \mathrm{y}),{ }^{73} \mathrm{Br}\left(t_{1 / 2}=3.4 \mathrm{~min}\right),{ }^{74} \mathrm{Br}^{m}\left(t_{1 / 2}=46 \mathrm{~min}\right)$, and ${ }^{75} \mathrm{Br}$ $\left(t_{1 / 2}=97 \mathrm{~min}\right)$ were produced on-line using the reaction of $150-\mathrm{MeV}{ }^{28} \mathrm{Si}$ on an ${ }^{54} \mathrm{Fe}$ target which formed part of the FEBIAD ion source of the Daresbury On-Line Isotope Separator (DOLIS). After acceleration to $60 \mathrm{keV}$, the selected bromine ions were implanted into a polycrystalline iron foil soldered to the cold finger of the dilution refrigerator and studied at temperatures down to $8 \mathrm{mK}$. Production rates were low for the lighter isotopes, but well resolved gamma spectra were recorded in four $\mathrm{Ge}(\mathrm{Li})$ detectors, two in both the axial and equatorial positions. The detector to source distance was $8 \mathrm{~cm}$. The iron foil was polarized by a 0.7-T magnetic field in a direction parallel to its surface and perpendicular to the implanted beam.

\section{DATA ANALYSIS}

The implanted $\mathrm{Br}$ nuclei are oriented at low temperatures through the large magnetic hyperfine interaction that they experience in an iron host lattice. The resulting angular distribution of gamma radiation from such nuclei is given by

$W(\theta)=1+\sum f_{i} \sum Q_{k} B_{k}\left(\mu B_{i} / I k T\right) U_{k} A_{k} P_{k}(\cos \theta)$,

the symbols having their usual meaning [9]. The index $i$ 
labels the various lattice sites which may be occupied by the implanted nuclei. The strength of the magnetic hyperfine interaction $\mu B_{i} / I$, and in particular the magnetic moment $\mu$ may then be determined from the temperature dependence of the anisotropy of the gamma-ray angular distribution, defined as $[W(0) / W(90)-1] \%$.

Within certain model limitations this interaction may be extracted from the data with relatively little dependence upon the fractional populations $f_{i}$ which, at moderately low temperatures, largely determine the absolute magnitude of the anisotropy but not its temperature dependence. This point will be discussed in more detail below. To facilitate the data analysis, two different models describing the lattice site occupation of the bromine nuclei in the iron matrix will be assumed. The first mod$\mathrm{el}$, henceforth known as model 1 , is that most applicable to soluble implants where a fraction $f$ of nuclei experience the full substitutional hyperfine field $B_{\text {sub }}$, while the remaining $1-f$ occupy lattice sites with zero hyperfine field. As an alternative, and to account in a simple way for the relative insolubility of bromine in iron, we consider a second model, hereafter referred to as model 2 . Here, as before, a fraction $f$ of nuclei experience the full substitutional hyperfine field $B_{\text {sub }}$, but now it is assumed that the remaining $1-f$ occupy a second lattice site subject to a single nonzero hyperfine field $B_{\text {low }}$.

Herzog et al. [10], using NMR-ON techniques for ${ }^{82} \mathrm{Br}$, have measured the substitutional magnetic hyperfine field of $\mathrm{BrFe}$ to be $B_{\text {sub }}=+81.38(6) \mathrm{T}$. Using this field it was found that the magnetic moment extracted from a model 1 fit to the integral LTNO data, as obtained from a low temperature implanted sample, differed by $10 \%$ from the known moment. A satisfactory fit could, however, be made using model 2 with $B_{\text {low }}= \pm 26$ (2) T. A subsequent study for both room and low temperature implanted samples over a wide range of doses showed that it was generally necessary to invoke model 2 in order to reproduce the magnetic moment [11]. The variation in the value of $B_{\text {low }}$ extracted from the various runs was no greater than would be expected from the statistical error of the value quoted above. No further complexity of site distribution can be meaningfully tested by the integral LTNO method. Extensive channeling studies by Alexander et al. [12], in the $\mathrm{Br} \underline{\mathrm{Fe}}$ system at room temperature and relatively high implantation dose, support this multiple site picture. They concluded that there was indeed a second, well defined, nonsubstitutional site and that this location was independent of the local bromine concentration over a wide range. Building upon this work, Callaghan et al. [13] performed an integral LTNO experiment on ${ }^{82} \mathrm{BrFe}$ in order to evaluate the two hyperfine fields. On the reasoned assumption that the second field contributed little to the experimental anisotropy and could therefore be neglected, they determined the substitutional hyperfine field to be $\pm 84(12) \mathrm{T}$ with an associated population of $36(5) \%$. Thus even using the crude model 1 they were able to predict the substitutional hyperfine field to within $15 \%$.

On the basis of these results it can be expected that the model uncertainties will only influence the extracted moments at the level of $10-15 \%$. Fitted moments obtained from both of the above models will be quoted in this work. The values ultimately adopted, however, will be those of model 2 after assigning uncertainties which reflect the model site distribution uncertainties as well as the statistical uncertainties.

\section{RESULTS}

\section{A. Results for ${ }^{76,77} \mathrm{Br}^{8}$}

Both of these nuclei were studied prior to the publication of Refs. $[10,11]$ and so it was not anticipated that the shortcomings of model 1 would be significant. Since the magnetic moment of ${ }^{76} \mathrm{Br}\left(1^{-}\right)$had already been measured [14] it was considered sufficient, and indeed desirable, to perform a less full temperature dependence for ${ }^{76} \mathrm{Br}$ in order to extract the $U_{2} A_{2}$ coefficients which were not known in advance. To this end a $\mathrm{PrNi}_{5}$ demagnetization stage was used to cool the nuclei to $2 \mathrm{mK}$, as measured by a ${ }^{60} \mathrm{CoNi}$ thermometer, which resulted in the saturation of the gamma-ray anisotropies. Unfortunately, these anisotropies were generally rather small, even close to saturation. Of the strongest lines, only the 1130-, 1854-, and 2951-keV transitions gave anisotropies with sufficiently favorable proportional errors to produce reasonable temperature dependences. The data on the 1130- and 2951-keV transitions are illustrated in Fig. 1.

Within the framework of model 1 a fit was performed to the experimental axial-equatorial anisotropies with both $\mu B_{\text {sub }}$ and $f U_{2} A_{2}$ as variable parameters. From
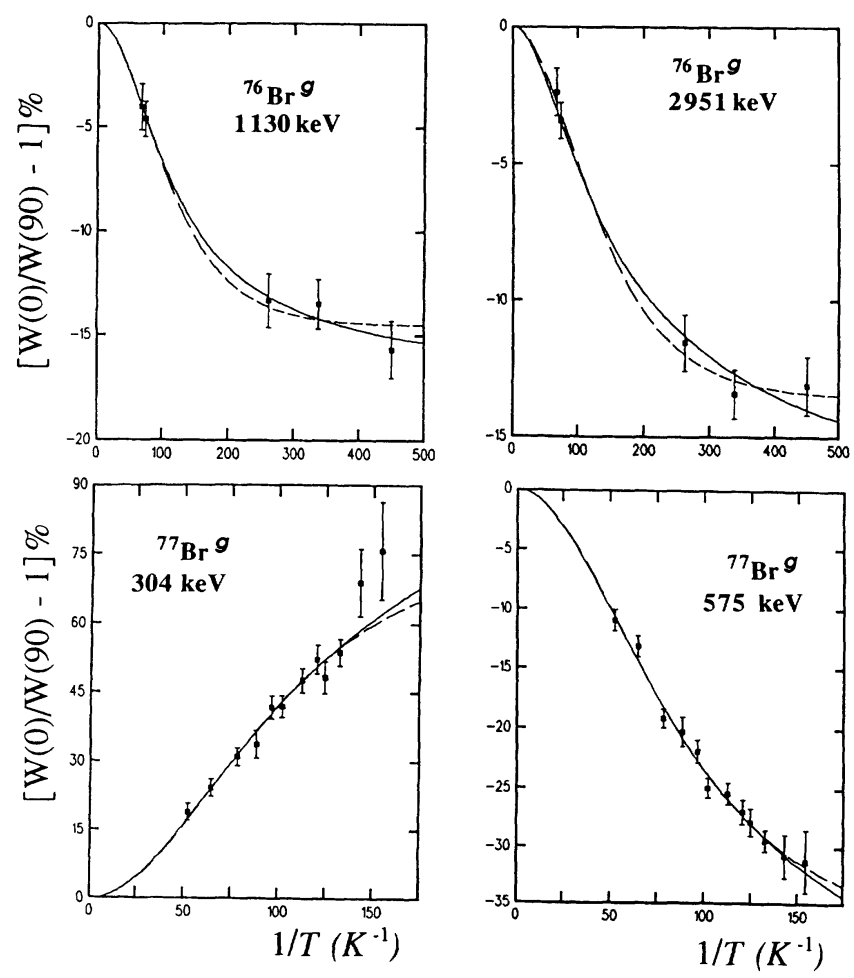

FIG. 1. The temperature dependence of the anisotropies of the 1130- and $2950-\mathrm{keV} \gamma$ rays in the decay of ${ }^{76} \mathrm{Br}^{8}$ and the anisotropies of the 304- and $575-\mathrm{keV} \gamma$ rays in the decay of ${ }^{77} \mathrm{Br}^{8}$. Both model 1 (dashed) and model 2 (solid) fitted curves are shown for comparison. 
these data the average hyperfine interaction was found to be $\mu B_{\text {sub }}=35.0(25) \mu_{N} T$. This is to be compared with the expected value (corrected for applied field) of $\mu B_{\text {sub }}=45.16(3) \mu_{N} T$.

This $25(6) \%$ difference highlights once again the failure of model 1 to adequately describe the $\mathrm{Br} \underline{\mathrm{Fe}}$ system as first noted by Herzog et al. [10]. Following their lead therefore, the more realistic model 2 is invoked. Here, with $\mu$ required to reproduce the known value, $U_{2} A_{2}, f$, and $B_{\text {low }}$ remain as variable parameters. In order to reduce the number of unknowns, $B_{\text {low }}$ was taken to be $26(2) \mathrm{T}$ as discussed previously. In this way we obtain a site distribution parameter of $f=58(9) \%$.

In order to understand how model 2 is able to correctly reproduce the substitutional hyperfine interaction it is instructive to consider the individual contributions from the various lattice sites, as shown in Fig. 2. The anisotropy data from the 1130-, 1854-, and 2951-keV transitions have been inverted to yield $\sum_{i} f B_{2} U_{2} A_{2}$ and then normalized to the 14-mK temperature points, which for this purpose have been combined, and finally summed. The resulting data therefore represent the relative orientation of the ${ }^{76} \mathrm{Br}$ nuclei as a function of temperature. The orientation predicted by model 1 , shown dashed, arises solely from the hyperfine interaction of nuclei in substitutional sites. It is clear that the true substitutional hyperfine interaction, $\mu B_{\text {sub }}=45.2 \mu_{N} T$, increasingly underestimates the experimental orientation as the temperature is reduced. This gap between the observed orientation and that due to the fully substitutional lattice site may be bridged by introducing a second lattice site which experiences a nonzero hyperfine interaction. The individual contributions of the substitutional and low field sites, weighted by their relative populations, to the total model 2 orientation are illustrated by the solid lines. The discrepancy between the magnetic moments extracted using the two models may be attributed to the proportion of the total orientation arising from this second nonsub-

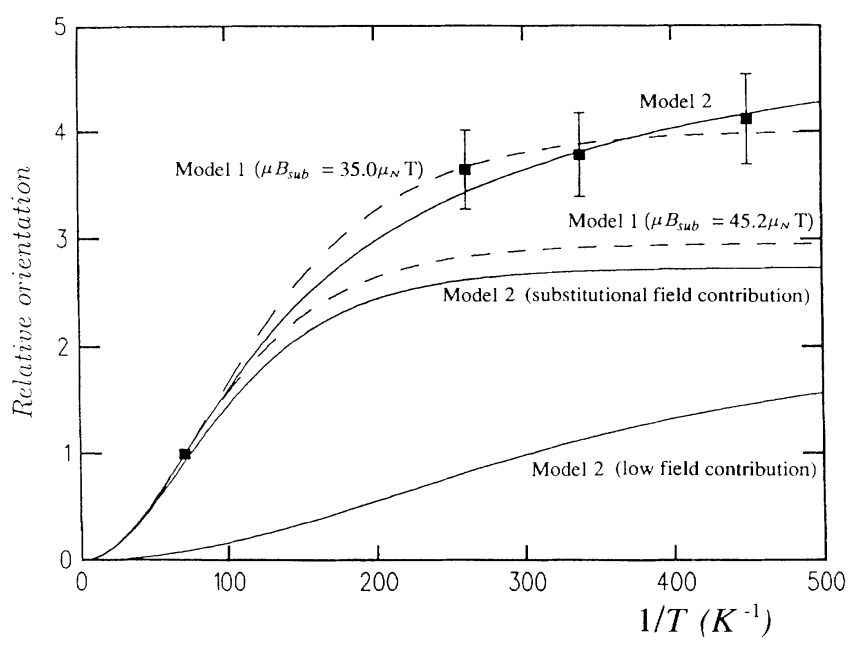

FIG. 2. The contributions to the total orientation from the model lattice sites. The data are taken from transitions in the decay of ${ }^{76} \mathrm{Br}^{m, g}$. The model 1 and model 2 fitted curves are distinguished by dashed and solid lines, respectively. stitutional hyperfine interaction. In the present case this proportion is as high as $35 \%$ at the lowest temperature datum, which results in the large disparity between the two extracted moments. The magnetic moments of the other nuclei studied in this work have all been extracted from data taken at temperatures above $6 \mathrm{mK}$, where the relative contribution from the second field site is greatly reduced. The model differences and hence the ambiguity in the extracted moment will therefore be correspondingly smaller.

Having determined the site distribution parameter $f=58(9) \%$ of ${ }^{76} \mathrm{Br} \underline{F e}$ it is assumed that the same value may be applied to the case of ${ }^{77} \mathrm{Br}$. This is justified since both were implanted under the same conditions and with similar dosage. Further, it is likely that the experimental error in $f$ is sufficiently large to take account of any minor variations between the samples.

In contrast to ${ }^{76} \mathrm{Br}^{g}$, the magnetic moment of ${ }^{77} \mathrm{Br}\left(\frac{3}{2}^{-}\right)$ had not previously been measured and therefore we performed a full temperature dependence down to $6 \mathrm{mK}$ as deduced from the anisotropy of a ${ }^{60} \mathrm{CoCo}$ thermometer.

In order to analyze these data, we take the model 1 variable parameters to be $\mu$ and $f U_{2} A_{2}$, and the model 2 variable parameters to be $\mu$ and $U_{2} A_{2}$, considering $f$ and $B_{\text {low }}$ as known. It should be noted that the site distribution parameter $f$ is not the same in the two models. The temperature dependences of the 304- and 575-keV transitions are shown in Fig. 1. Using data from the ten strongest transitions we obtain an average model 1 moment of $\mu=0.82(3) \mu_{N}$, while for model 2 we obtain a moment of $\mu=0.92(5) \mu_{N}$. Notice that as a result of the higher temperatures there is only an $11(7) \%$ disagreement between the two models. Comparing the $\chi^{2}$ values of the two sets of model fits, which are to all intents and purposes identical, we find that even with data of such relatively good quality the integral LTNO method is unable to distinguish between the two models. In recent studies in which ${ }^{77} \mathrm{Br}$ has been studied by NMR methods, a more precise value of $0.9738(5) \mu_{N}$ has been reported, a value at the edge of the uncertainty of the integral LTNO method [15].

\section{B. Results for ${ }^{72-75} \mathrm{Br}$}

These isotopes were implanted on-line into a cold iron foil at the DOLIS-COLD facility where temperatures as low as $8 \mathrm{mK}$ were achieved, as deduced from the anisotropy of a ${ }^{57} \mathrm{CoFe}$ thermometer. Unlike the previous case, there is no control isotope with a known moment with which to determine $f$. Therefore within the framework of model 2 there are at least four unknown parameters, $\mu$, $f, U_{\lambda} A_{\lambda}$, and $B_{\text {low }}$. In order to be able to extract reliable values for the magnetic moment from the fitting procedure it is desirable to reduce this number to two. Since the $U_{\lambda} A_{\lambda}$ coefficients are not generally known a priori, they must be considered as variable parameters. Therefore not only $B_{\text {low }}$ but also a range of values for the site distribution parameter $f$ is assumed.

The variation in $f$ as a function of implantation conditions of ${ }^{82} \mathrm{Br} \underline{\mathrm{Fe}}$ has also been studied [11]. It is apparent from this work that the value of $f$ is considerably depen- 
dent upon implantation dose and also the method of production. However, the values for different on-line implantations were all found to lie within $58 \% \leq f \leq 75 \%$. Although some dependence upon the method of preparation of the iron foil might also be expected, this range will be considered to be valid for the present work. Since all iron foils used in the present experiments were prepared in an identical manner, the dependence upon the method of preparation of the iron foils will not lead to any problems with internal self-consistency. Thus the likely variation in $f$ will be spanned if a maximum value of $75 \%$, model $2 \mathrm{a}$, and a minimum value of $58 \%$, model $2 \mathrm{~b}$, is taken. It should be noted that the choice of $58 \%$ as the minimum value for the site distribution parameter in online samples coincides with the value deduced for the offline prepared samples ${ }^{76,77} \mathrm{Br}$. That the site distribution parameter is probably lower for off-line than for on-line prepared samples is apparent from the data of Ref. [11] and is in line with our work on implanted $\mathrm{BrFe}$ [13].

\section{Results for ${ }^{75} \mathrm{Br}$}

The presence in the DOLIS output of an inactive contaminant beam at this mass required that the ${ }^{75} \mathrm{Br}$ source be built up by implanting for $2.5 \mathrm{~h}$ at approximately 50 $\mathrm{mK}$. The procedure was then followed by a period of cooling down to temperatures of $9 \mathrm{mK}$ with the isotope separator closed off. The cycle was repeated several times.

With the exception of the $377-\mathrm{keV}$ transition, which gave data of good statistical accuracy, the resulting anisotropies were rather small. The temperature dependence of this peak is illustrated in Fig. 3. In addition, and uniquely among all the isotopes studied, the $U_{2} A_{2}$ coefficient can be calculated for this transition with sufficient accuracy to enable some constraint to be placed on the fitting procedure. The use of the previously known mixing ratio for the $377-\mathrm{keV}$ transition, $\delta(377)=-0.73(45) \quad[16]$, leads to the inequality $+0.62 \leq U_{2} A_{2} \leq+0.81$. The model 2 fitted $U_{2} A_{2}$ coefficient is compatible with this result to within one standard deviation for all site distribution parameters $f \geq 44 \%$, which greatly exceeds the likely range of variation given above. Therefore the limiting values $75 \%$ (model $2 \mathrm{a}$ ) and $58 \%$ (model $2 \mathrm{~b}$ ) are retained, and with $f$ thus fixed, the inequality for $U_{2} A_{2}$ is used to limit the error on the fitted moment. In this way we find moments of $0.73(9) \mu_{N}, 0.75(15) \mu_{N}$, and $0.78(17) \mu_{N}$ for models $1,2 \mathrm{a}$, and $2 \mathrm{~b}$, respectively. [For comparison we give the unconstrained values of $0.73(9) \mu_{N}, 0.75(19) \mu_{N}$, and $0.78(24) \mu_{N}$, respectively. Of course in model 1 this constraint has no effect on the fitted moment and merely serves to provide the inequality $f \geq 53 \%$.]

Note that despite the large difference between the site distribution parameters of models $2 \mathrm{a}$ and $2 \mathrm{~b}$ there is only a very slight difference in the fitted moments.

\section{Results for ${ }^{74} \mathrm{Br}^{\mathrm{m}}$}

The heavy ion reaction of $150-\mathrm{MeV}{ }^{28} \mathrm{Si}$ on ${ }^{54} \mathrm{Fe}$ preferentially produced the high-spin isomer of ${ }^{74} \mathrm{Br}$. The pro-
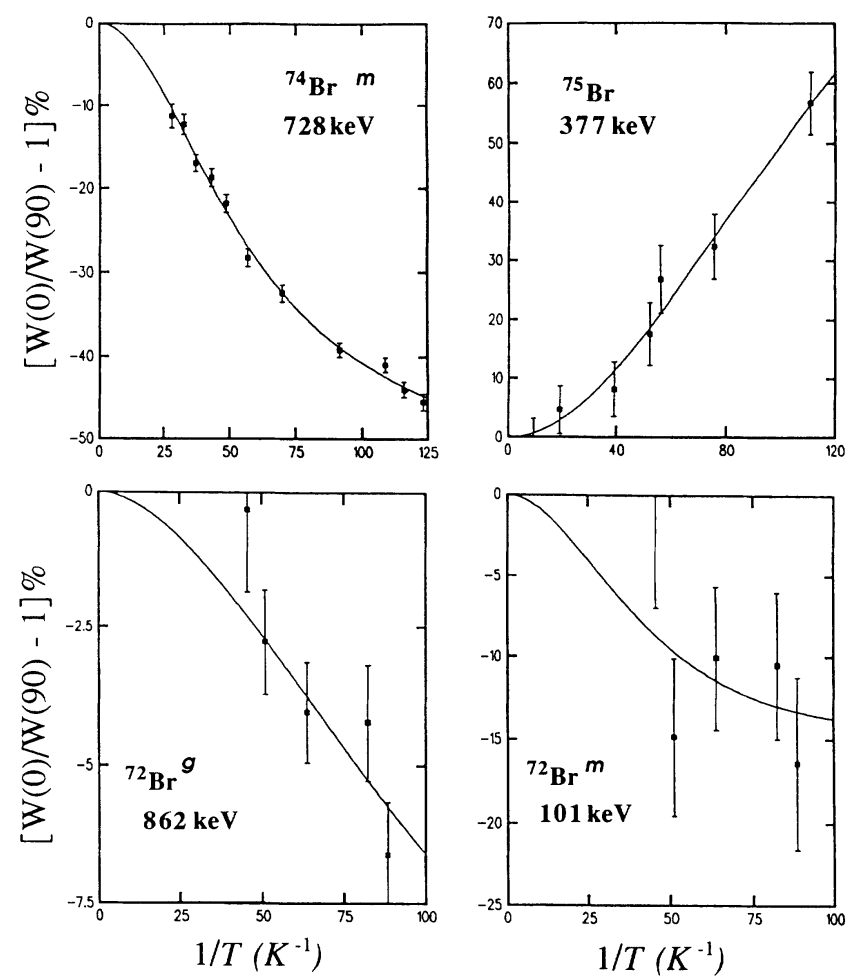

FIG. 3. The temperature dependance of the anisotropies of the 377-, 728-, 862-, and $101-\mathrm{keV} \gamma$ rays in the decays of ${ }^{75} \mathrm{Br}$, ${ }^{74} \mathrm{Br}^{m},{ }^{72} \mathrm{Br}^{m}$, and ${ }^{72} \mathrm{Br}^{g}$, respectively. The model 1 and model 2 fitted curves coincide.

portion of the ground state present, as deduced from the intensity ratio of the $615-$ to $634-\mathrm{keV}$ transitions, was less than $5 \%$ and therefore essentially negligible. Large anisotropies with small statistical errors were observed for the $728-\mathrm{keV}$ transition, shown in Fig. 3, and also for the 615-, 634-, 839-, 1201-, 1250-, and 1269-keV transitions.

The isomeric spin has been deduced by atomic beam resonance methods to be $I=4$ [17], although the assignment of negative parity to this state, made on the basis of proposed first unique forbidden $\beta^{+} / \mathrm{EC}$ decays to levels in ${ }^{74} \mathrm{Se}$, is rather tentative. Partly as a result of this condition, but mainly due to the complex decay scheme, no $U_{\lambda} A_{\lambda}$ coefficients are known or can be calculated $a$ priori. Further, due to the relatively large parent spin, these coefficients involve two terms corresponding to $\lambda=2$ and 4 .

As a first step towards dealing with this extra parameter the data were fitted to model 2 a ignoring fourth rank terms. The weighted mean of these moments, for the seven transitions given above, is $1.64(5) \mu_{N}$ for which at $10 \mathrm{mK}$ the ratio of the high field orientation parameters is $B_{4}\left(B_{\text {sub }}\right) / B_{2}\left(B_{\text {sub }}\right) \sim 30 \%$. Although this ratio is likely to become smaller when allowance is made for the relative effects of the two $U_{\lambda} A_{\lambda}$ terms, the fourth rank terms possibly constitute a sizable effect. We therefore also include these terms as a third variable in fits to the temperature dependences of the statistically dominant 634- and $728-\mathrm{keV}$ transitions, from which we obtain moments of $1.63(6) \mu_{N}, 1.59(8) \mu_{N}$, and $1.77(11) \mu_{N}$ for models $1,2 \mathrm{a}$, and $2 b$, respectively. Once again the relatively small 
difference between the model $2 \mathrm{a}$ and $2 \mathrm{~b}$ moments demonstrates their limited sensitivity to the assumed site distribution parameter. Recent NMR studies have yielded a somewhat more precise value of $1.82(1)$ that is independent of the assumptions about site occupancy [15].

\section{Results for ${ }^{73} \mathrm{Br}$}

Anisotropies were observed for 16 of the strongest transitions in the decay of ${ }^{73} \mathrm{Br}$. These are listed in Table I. The anisotropy of the ${ }^{57} \mathrm{CoFe}$ thermometer corresponds to an average temperature of $12 \mathrm{mK}$. All the ${ }^{73} \mathrm{Br}$ effects are consistent with zero orientation. Since the iron foil in which ${ }^{73} \mathrm{Br}$ was studied was the same as that used to successfully orient ${ }^{72,74} \mathrm{Br}^{m}$ in the same on-line run, there can be no possibility of a non-nuclear origin for this lack of anisotropy.

The ground-state spin of ${ }^{73} \mathrm{Br}$ had been limited to $\left(\frac{1}{2}, \frac{3}{2}, \frac{5}{2}\right)^{-}$as a consequence of its allowed $\beta^{+} / \mathrm{EC}$ decay to the $26-\mathrm{keV} \frac{3}{2}^{-}$level in ${ }^{73} \mathrm{Se}[18]$. On the basis of systematics in the neighboring odd- $A$ isotopes this spin has often been taken as $\frac{3}{2}^{-}$. However, the lack of anisotropy is at variance with this hypothesis.

If the ground state were $\frac{3}{2}^{-}$and had a moment similar to that of ${ }^{75} \mathrm{Br}$ then the relaxation time would be of the order of $5 \mathrm{~s}$ (see below). When compared to the 3.4-m half-life it is clear that incomplete relaxation cannot be responsible for reducing the orientation to the virtually zero levels observed.

It must therefore be concluded that the lack of observed anisotropy is due to the presence of one or more unoriented spin $-\frac{1}{2}$ levels. The transitions in ${ }^{73} \mathrm{Se}$ originate from many different levels which are independently populated in the decay. A recent spectroscopic study of the decay of ${ }^{73} \mathrm{Br}$ [19] has provided spin assignments for some of the levels assuming that the parent spin is $\frac{3}{2}^{-}$. While most of these are tentative it is clear that not all of the levels which give rise to the observed gamma transitions are likely to be of spin $\frac{1}{2}$. It is therefore most probable

TABLE I. The gamma-ray angular distribution anisotropies observed in the decay of oriented ${ }^{73} \mathrm{Br}$ at $12 \mathrm{mK}$.

\begin{tabular}{crc}
\hline \hline Energy $(\mathrm{keV})$ & $A(\%)(\sigma)$ & Anisotropy/ \\
\hline 65 & $0.6(0.6)$ & 1.0 \\
275 & $0.8(4.9)$ & 0.2 \\
336 & $0.8(1.6)$ & 0.5 \\
374 & $-1.6(5.2)$ & -0.3 \\
401 & $-0.8(2.0)$ & -0.4 \\
490 & $7.0(8.4)$ & 0.8 \\
540 & $-0.3(3.7)$ & -0.1 \\
550 & $4.1(7.3)$ & 0.6 \\
615 & $-6.5(4.4)$ & -1.5 \\
639 & $-6.5(10.3)$ & 0.6 \\
700 & $1.9(1.4)$ & 1.4 \\
788 & $-10.0(11.0)$ & -0.9 \\
849 & $-0.3(1.1)$ & -0.3 \\
870 & $6.6(4.8)$ & 1.4 \\
914 & $3.1(2.9)$ & 1.1 \\
931 & $1.5(1.5)$ & 1.0 \\
\hline \hline
\end{tabular}

that at least a few of the relevant levels in ${ }^{73} \mathrm{Se}$ should be capable of orientation. In principle the lack of orientation might also be due to unfortunate values of the gamma multipole mixing ratios which lead to near zero angular distribution coefficients. This possibility is also inherently unlikely to be simultaneously true for several transitions. Two levels in ${ }^{73} \mathrm{Se}$ (other than the ground state) are in fact known to have spins other than $\frac{1}{2}$ [18]. Unfortunately neither figure prominently in this work. The 151-keV $\frac{5}{2}^{-}$level gives rise only to the $126-\mathrm{keV}$ transition which lies between the $122-$ and $136-\mathrm{keV}$ peaks of the thermometer, ${ }^{57} \mathrm{CoFe}$. Although the triplet is resolved, the background is discontinuous making its subtraction difficult and ambiguous. However, a crude manual background subtraction showed no significant anisotropy for this transition. The second level at $26 \mathrm{keV}$ $\left(\frac{3}{2}^{-}, 40 \mathrm{~min}\right)$ is isomeric and therefore orients in its own right.

The decay of the high-spin states of ${ }^{73} \mathrm{Br}$ has recently been studied by Heese et al. [20,21]. They have been able to provide a satisfactory interpretation of the level structure of ${ }^{73} \mathrm{Br}$ using a $\frac{1}{2}^{-}$ground-state spin and parity assignment. Moreover, another recent study of the levels of ${ }^{71} \mathrm{Br}$ has provided evidence for a $\frac{5}{2}^{-}$ground state and a $\frac{1}{2}^{-}$level at $9 \mathrm{keV}$ in that nuclide [22]. Consequently, we conclude that it is in fact the parent nucleus, ${ }^{73} \mathrm{Br}$, which has a ground-state spin $\frac{1}{2}^{-}$. Although such an assignment differs from the spin- $\frac{3}{2}^{-}$ground states of all the heavier odd isotopes up to ${ }^{87} \mathrm{Br}$, it is fully consistent with all known features of the decay, and with the decay of the higher-spin levels and the structure of ${ }^{71} \mathrm{Br}$.

\section{Results for $^{72} \mathrm{Br}^{8, m}$}

The anisotropy of the strongest line in the decay of ${ }^{72} \mathrm{Br}^{g}$, at $862 \mathrm{keV}$, is shown in Fig. 3. In this nuclide, there occurs the added complication that the relatively short half-life $(1.3 \mathrm{~m})$ may not allow sufficient time for the attainment of thermal equilibrium. Relaxation measurements by Herzog [10] on ${ }^{82} \mathrm{BrFe}$ have yielded the result $g^{2} C_{k}=0.032(2)$, where $g$ is the spin $g$ factor and $C_{k}$ the Korringa constant [9]. The product $g^{2} C_{k}$ is an isotope independent quantity which allows a self-consistent calculation to be performed on the data of ${ }^{72} \mathrm{Br}$, taking into account the effects of imperfect relaxation on the observed anisotropies. The fitted line in Fig. 3 is calculated for a moment of $0.55(21) \mu_{N}$, for which the anisotropy attenuation at $12.5 \mathrm{mK}$ is about $15 \%$. This result is virtually independent of the choice of the model, the small differences lying well within the statistical uncertainties.

Finally, we consider the $101-\mathrm{keV}$ transition from the 10.1-s isomeric state, to the ground state, which was assumed to be a $1^{-}$to $3^{+}$transition by Garcia-Bermudez et al. [23]. This spin parity change is confirmed by present $\gamma$-conversion electron coincidence measurements [24]. These data yield a conversion coefficient $\alpha_{K}=1.4(3)$, implying an almost pure $M 2$ transition, with less than $13 \% E 3$ admixture. Analysis of the NO data, shown in Fig. 3, was difficult owing to the large Compton background associated with the photopeak and the shape 
of the temperature dependence was ill defined, resulting in a fitted moment of $1.3_{-0.5}^{+10.0} \mu_{N}$. Again the model dependence is well within the uncertainties. Taking the measured anisotropy of $-11(3) \%$ at $15.1 \mathrm{mK}$ and assuming the maximum magnitudes for $f$ and $A_{2}$ of $100 \%$ and -0.2778 , respectively, then we can set a probable lower limit on the moment of $0.7 \mu_{N}$.

\section{EXPERIMENTAL CONCLUSIONS}

LTNO measurements on the light bromine nuclei have yielded the nuclear magnetic dipole moments of ${ }^{72} \mathrm{Br}^{g, m}$, ${ }^{74} \mathrm{Br}^{m},{ }^{75} \mathrm{Br}$, and ${ }^{76,77} \mathrm{Br}^{g}$. In addition, the ground-state spin of ${ }^{73} \mathrm{Br}$ has been established as $\frac{1}{2}^{-}$.

The experimental data have been analyzed within the framework of the two models described in Sec. III. The results of this analysis are summarized in Table II. It has been shown, both here for the case of ${ }^{76} \mathrm{Br}$ and by Herzog et al. $[10,11]$ for the case of ${ }^{82} \mathrm{Br}$, that model 1 fails to yield the correct moment. Therefore it is necessary to adopt the more flexible model 2. This approach requires an extra parameter, $B_{\text {low }}$ and also causes $f$ and $U_{\lambda} A_{\lambda}$ to become independent. Since the $U_{\lambda} A_{\lambda}$ coefficients are generally not known a priori for the isotopes studied, a limited range of values for $f$ has been assumed in order to reduce the number of free parameters to be extracted from the experimental data.

For ${ }^{77} \mathrm{Br}, f$ is taken to be the same as that deduced from the data on the control isotope ${ }^{76} \mathrm{Br}$ since the two were produced under similar conditions. For ${ }^{72-75} \mathrm{Br}$ no such control exists and it is necessary to adopt a range of likely values for $f$, taken to be $58 \% \leq f \leq 75 \%$ (models $2 \mathrm{a}$ and $2 \mathrm{~b}$, respectively). It has been shown that within this range the model 2 extracted moments have only a limited sensitivity to the value of $f$.

In addition, it is in principle necessary to consider also the effects of a variable low field $B_{\text {low }}$, since $B_{\text {low }}$ need not correspond to a well defined field but may, instead, represent some average over many fields. However, the work of Herzog et al. [10,11] has given some evidence that $B_{\text {low }}$ varies by no more than $2 \mathrm{~T}$ between different warm or cold implantations The value of $f$, on the other hand, varied by $20 \%$ between warm and cold implantations and so it is clear that $f$ and not $B_{\text {low }}$ should be treated as the variable parameter.

In view of the proven inability of model 1 to correctly describe the experimental data the most reliable moments are taken to be those derived from model 2 . In the case of ${ }^{75} \mathrm{Br}$, where both models $2 \mathrm{a}$ and $2 \mathrm{~b}$ have been applied, the mean of the two results is taken with errors chosen so as to encompass the range of values emerging from the two models. Effectively this procedure corresponds to the assumption that $f=67(9) \%$. The resulting increase in the uncertainty is relatively modest despite realistically reflecting the site distribution uncertainties. These moments along with the values from NMR are listed in Table II. The more precise values are of assistance in giving credence to the integral measurements and do not change significantly the nuclear structure information that can be derived from the whole set of data.

\section{DISCUSSION}

\section{A. The odd-mass Br nuclides}

The odd- $A$ bromine nuclei ${ }^{75-87} \mathrm{Br}$, with atomic number 35, are all assigned to have ground-state spins of $\frac{3}{2}^{-}$. In-beam spectroscopic studies of ${ }^{73} \mathrm{Br}[3,20,21,25,26]$ have revealed a band sequence which bears a remarkable resemblance to the ground-state band of ${ }^{75} \mathrm{Br}$. However, the last stretched $E 2$ transition terminates not on the ground state but instead on an excited level at $178 \mathrm{keV}$ which has therefore been assigned as $\frac{3}{2}^{-}$. Recent data on

${ }^{71} \mathrm{Br}$ show a similar situation with the band terminating at a level at $207 \mathrm{keV}[22,27]$. From Fig. 4 it can be seen that for prolate nuclei the 35 th proton is indeed expected to occupy the [301] $\frac{3}{2}$ orbital for quadrupole deformations up to $\varepsilon<0.22$ and the [312] $\frac{3}{2}$ orbital at larger deformations in the range $0.30<\varepsilon<0.40$. As these levels are associated with theoretical magnetic dipole moments of approximately $+2.3 \mu_{N}$ and $+0.75 \mu_{N}$, respectively, the experimental magnetic moments of the $\frac{3}{2}^{-}$ground states give a clear signature of the orbitals involved and hence the magnitude of the nuclear deformation.

Because the orbitals of interest to the odd- $\boldsymbol{A}$ bromine ground states have the same component of angular momentum and can therefore intermix, the transition between high and low moment is not sharp, but instead has a definite deformation dependence. This variation of the moment with deformation is most sensitive in the region $0.22<\varepsilon<0.30$ where, with increasing deformation, the Fermi level shifts from the [301] $\frac{3}{2}$ to the [312] $\frac{3}{2}$ orbital

TABLE II. A summary of the fitted moments of ${ }^{72-77} \mathrm{Br}$.

\begin{tabular}{lccccc}
\hline \hline Nuclide & & \multicolumn{4}{c}{ Fitted moments } \\
& Model 1 & $\begin{array}{c}\text { Model 2a } \\
\text { Model 2b }\end{array}$ & $\begin{array}{c}\text { Adopted integral value } \\
(f=58 \%)\end{array}$ & NMR moment \\
\hline${ }^{77} \mathrm{Br}$ & $0.82(2)$ & & $0.92(5)$ & $0.92(5)$ & $0.9738(5)^{\mathrm{b}}$ \\
${ }^{76} \mathrm{Br}$ & $0.43(3)$ & & 0.55 & $\pm 0.55^{\mathrm{a}}$ & $0.5482(1)^{\mathrm{a}}$ \\
${ }^{75} \mathrm{Br}$ & $0.73(9)$ & $0.75(15)$ & $0.78(17)$ & $0.76(18)$ & $1.82(1)^{\mathrm{b}}$ \\
${ }^{74} \mathrm{Br}^{m}$ & $1.63(6)$ & $1.59(8)$ & $1.77(11)$ & $1.68(18)$ & \\
${ }^{72} \mathrm{Br}^{8}$ & $0.55(21)$ & $0.55(21)$ & $0.55(21)$ & $0.55(21)$ & $>7$ \\
${ }^{72} \mathrm{Br}^{m}$ & $>0.7$ & $>0.7$ & $>0.7$ & & \\
\hline \hline
\end{tabular}

${ }^{\text {a }}$ Reference [14].

${ }^{\mathrm{b}}$ Reference [15]. 


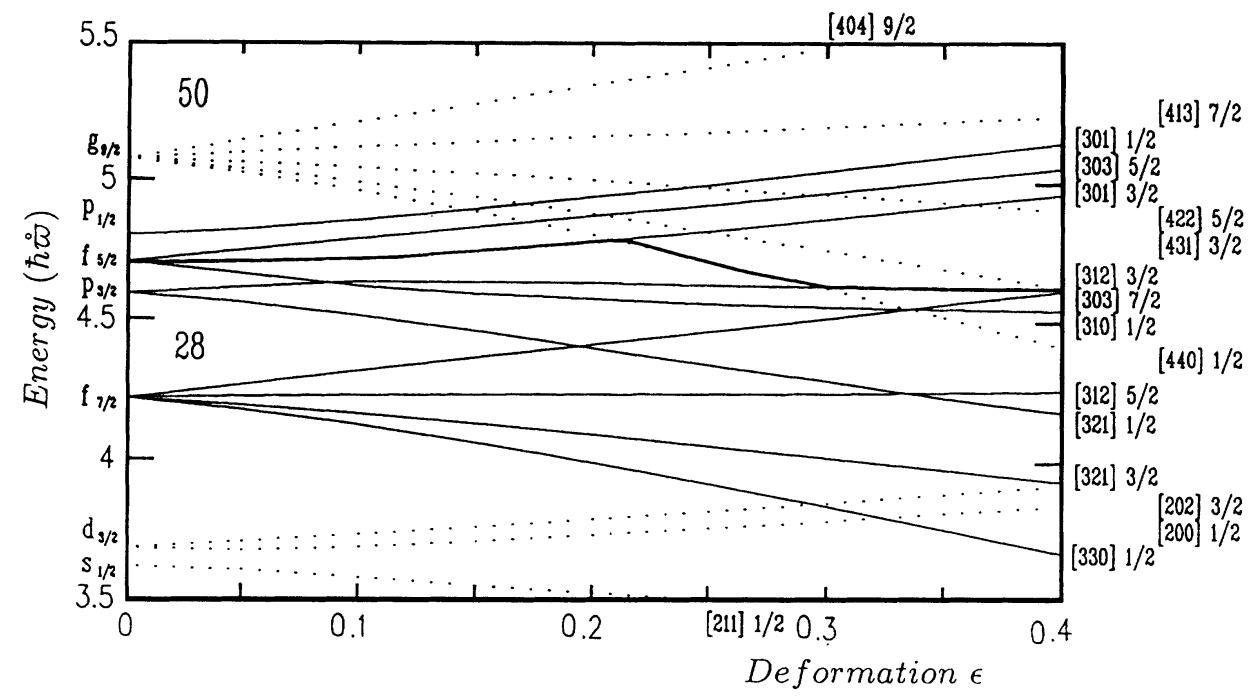

FIG. 4. Proton single-particle levels in the $A \sim 75$ region as a function of quadrupole deformation $\varepsilon$. The approximate position of the Fermi level in the $\mathrm{Br}$ nuclides is indicated by the heavy line. The Nilsson modified oscillator parameters $\kappa=0.068$ and $\mu=0.38$ are used. The dotted lines are the positive parity levels and the solid lines are the negative parity levels.

via the $[440] \frac{1}{2}$ state. As a consequence, the dominant single-particle contribution to the total wave function changes between the two configurations and is accompanied by a smooth and rather rapid drop in the magnetic moment from $+2.3 \mu_{N}$ to $+0.75 \mu_{N}$. The variation of the ground-state moment with deformation is illustrated in Fig. 5. Only the positions of the negative parity [301] $\frac{3}{2},[312] \frac{3}{2}$, and [303] $\frac{7}{2}$ bandheads, together with the $\frac{1}{2}^{-}$and $\frac{3}{2}^{-}$members of the [310] $\frac{1}{2}$ band, are shown.

To develop these ideas of ground-state evolution in $\mathrm{Br}$ isotopes, we performed two types of model calculation including low-energy states.

In the first type of calculation, the Lund particletriaxial rotor model [28] with the modified oscillator single-particle potential was used to fit experimental data

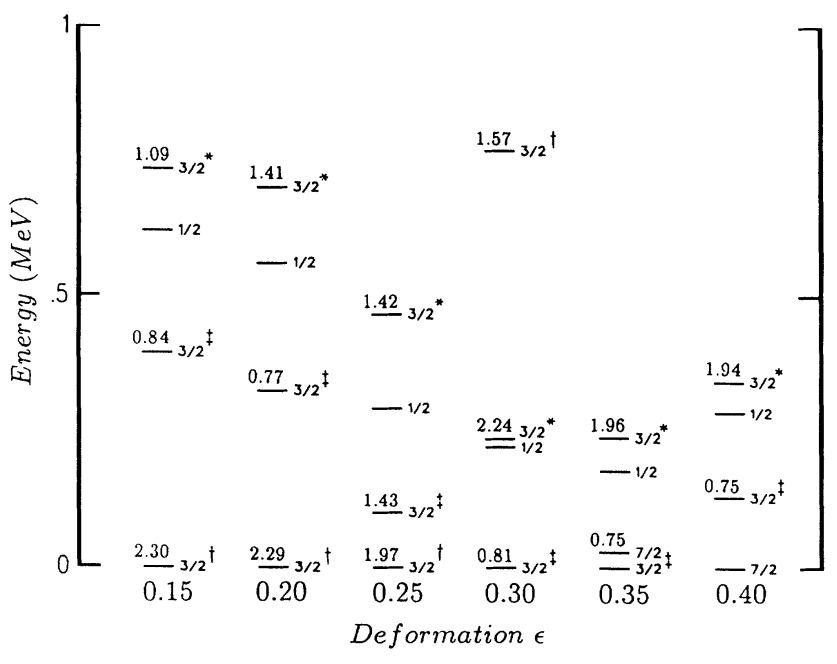

FIG. 5. Deformation dependence of the ground-state magnetic dipole moment in the odd- $\boldsymbol{A} \mathrm{Br}$ isotopes. The positions of the $[301] \frac{3}{2}^{\dagger},[312] \frac{3}{2}^{\ddagger}$, and [303] $\frac{7}{2}$ bandheads, together with the $\frac{1}{2}$ and $\frac{3}{2}(*)$ members of the [310] $\frac{1}{2}$ band, are indicated. The magnetic moments of these three lowest lying $\frac{3}{2}^{-}$states are given in $\mu_{N}$. on the energy levels and electromagnetic properties of negative parity low-energy levels in ${ }^{71-81} \mathrm{Br}$ in order to extract deformation parameters $\varepsilon_{2}$ and $\gamma$. The original version of this calculation $\operatorname{VMI}(\mathbf{A})$ [29] was performed taking the parameters $\kappa=0.068$ and $\mu=0.38$, equal for all oscillator shells. A variable moment of inertia was used with parameters $J_{0}$ and $C$ that were calculated using the experimental $2_{1}^{+}, 4_{1}^{+}$, and $2_{2}^{+}$level energies in the neighboring even-even Se nuclides [30]. We subsequently performed the same calculation with so-called "standard parameters" $\kappa$ and $\mu$ [31] both with a constant moment of inertia [28] (PTR-MO) and with a variable moment of inertia [VMI(S)]. In the $\operatorname{VMI}(\mathbf{S})$ calculation, $J_{0}$ was estimated using deformation extracted from total Routhian surfaces (TRS) (see below) and $C$ was obtained from the best fit to energy levels in odd- $A \mathrm{Br}$ isotopes. $\varepsilon_{2}$ and $\gamma$ were the only deformation parameters employed in calculations using the modified oscillator potential. The effect of including $\varepsilon_{4}$ on the quality of the fit was examined and found negligible.

In the second type of calculation, we used the particletriaxial rotor model with Woods-Saxon single-particle potential (PTR-WS) and a constant irrotational moment of inertia in order to calculate level energies and electromagnetic properties of ${ }^{71-81} \mathrm{Br}$ with deformation parameters $\beta_{2}, \beta_{4}$, and $\gamma$ extracted from TRS [32]. TRS were calculated using the deformed mean field approach with the Woods-Saxon potential, Strutinsky shell corrections, and a monopole pairing force [33].

In all of the above calculations, pairing was treated using the standard BCS approximation. In the determination of electromagnetic properties the effective $g_{s}$ factor was taken to be $0.65 g_{s}$ free. Quadrupole moments of the core were calculated macroscopically and $g_{R}=Z / A$ was used. No attenuation of the Coriolis matrix elements was applied. There were thus no variable parameters in the PTR-WS calculation, only two variable parameters, $\varepsilon_{2}$ and $\gamma$, in the PTR-MO and VMI(A) calculations, and three, $\varepsilon_{2}, \gamma$, and $C$ in the $\operatorname{VMI}(\mathbf{S})$ calculation.

The TRS calculations give collective minima for ${ }^{70-74} \mathrm{Se}$ and ${ }^{72-78} \mathrm{Kr}$ and the corresponding deformation 


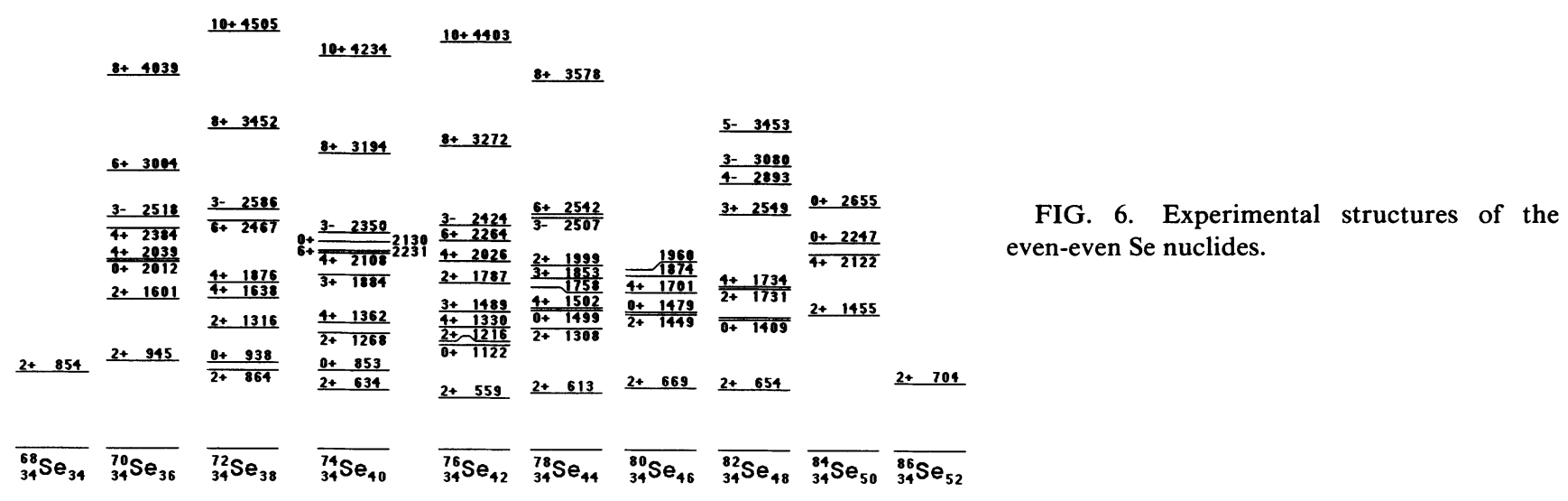

parameters $\beta_{2}, \beta_{4}$, and $\gamma$ as a function of rotational frequency $\omega$. The experimental energy level structure of these nuclides is shown in Figs. 6 and 7. For all these nuclei the calculations predict, for $\omega$ close to zero, coexisting prolate and oblate shapes having different deformations (see Table III). No collective minimum is predicted in heavier $\mathrm{Se}(A=76-80)$ and $\mathrm{Kr}(A=80-82)$. Using the parameters from the TRS calculations, we made calculations for ${ }^{71-75} \mathrm{Br}$ for both $\mathrm{Se}$ and $\mathrm{Kr}$ cores and ${ }^{77} \mathrm{Br}$ for a $\mathrm{Kr}$ core only. For ${ }^{79,81} \mathrm{Br}$, where no TRS data are available, we performed the PTR-WS calculation, fitting the deformation parameters to energy levels and the ground-state magnetic dipole and electric quadrupole moments.

Theoretical results are summarized in Table III showing the best fit deformation parameters from different calculations for odd- $A \mathrm{Br}$ and the TRS results for even-even $\mathrm{Se}$ and $\mathrm{Kr}$. Table IV displays experimental and calculated ground-state magnetic dipole and electric quadrupole moments together with $B\left(E 2 ; \frac{7}{2}^{-}\right.$to $\left.\frac{3}{2}^{-}\right)$and $B\left(E 2 ; \frac{13}{2}^{-}\right.$ to $\left.\frac{9}{2}^{-}\right)$values in the $\frac{3}{2}^{-}$yrast bands. Table $\mathrm{V}$ illustrates the calculated structure of the $\frac{3}{2}^{-}$bandhead in ${ }^{71-81} \mathrm{Br}$.

Our results point to a gradual increase in nuclear ground-state deformation from about $\varepsilon_{2}=0.20, \gamma=0$ in
${ }^{81} \mathrm{Br}$ to $\varepsilon_{2}=0.33, \gamma=0$ in ${ }^{75} \mathrm{Br}$. The yrast $\frac{3}{2}^{-}$prolate band in ${ }^{71,73} \mathrm{Br}$ still has strong deformation, $\varepsilon_{2}>0.30$. This trend is accompanied by a drop in the experimental magnetic dipole moment from $+2.3 \mu_{N}$ to $+0.75 \mu_{N}$ for ${ }^{75-81} \mathrm{Br}$ very well reproduced by theory (see Table IV). This trend indicates a transition of the single-particle Fermi level from the [301] $\frac{3}{2}$ to the [312] $\frac{3}{2}$ orbital. In Table $\mathrm{V}$ we see that the ground state of ${ }^{81} \mathrm{Br}$ is dominated by the $[301] \frac{3}{2}$ orbital, ${ }^{79} \mathrm{Br}$ is transitional, while the ground states of ${ }^{77,75} \mathrm{Br}$, the $178-\mathrm{keV}$ excited state of ${ }^{73} \mathrm{Br}$, and the $207-\mathrm{keV}$ excited state of ${ }^{71} \mathrm{Br}$ are almost entirely of [312] $\frac{3}{2}$ character. We note that the asymptotic quantum numbers are used in this work only to label particular Nilsson orbitals. Therefore the quoted results represent the relative contributions from the two Nilsson levels and do not necessarily reflect the exact singleparticle configurations.

The $\frac{5}{2}^{-}$ground state of ${ }^{71} \mathrm{Br}$ [22] and the proposed $\frac{1}{2}^{-}$ ground state in ${ }^{73} \mathrm{Br}$ appear to arise from a fundamentally different structure. As can be seen from Fig. 8, there is no orbital lying near the position of the Fermi surface in bromine which would give rise to such a ground state at any prolate deformation. In particular, the [310] $\frac{1}{2}$ orbital cannot be placed on the Fermi surface without significant

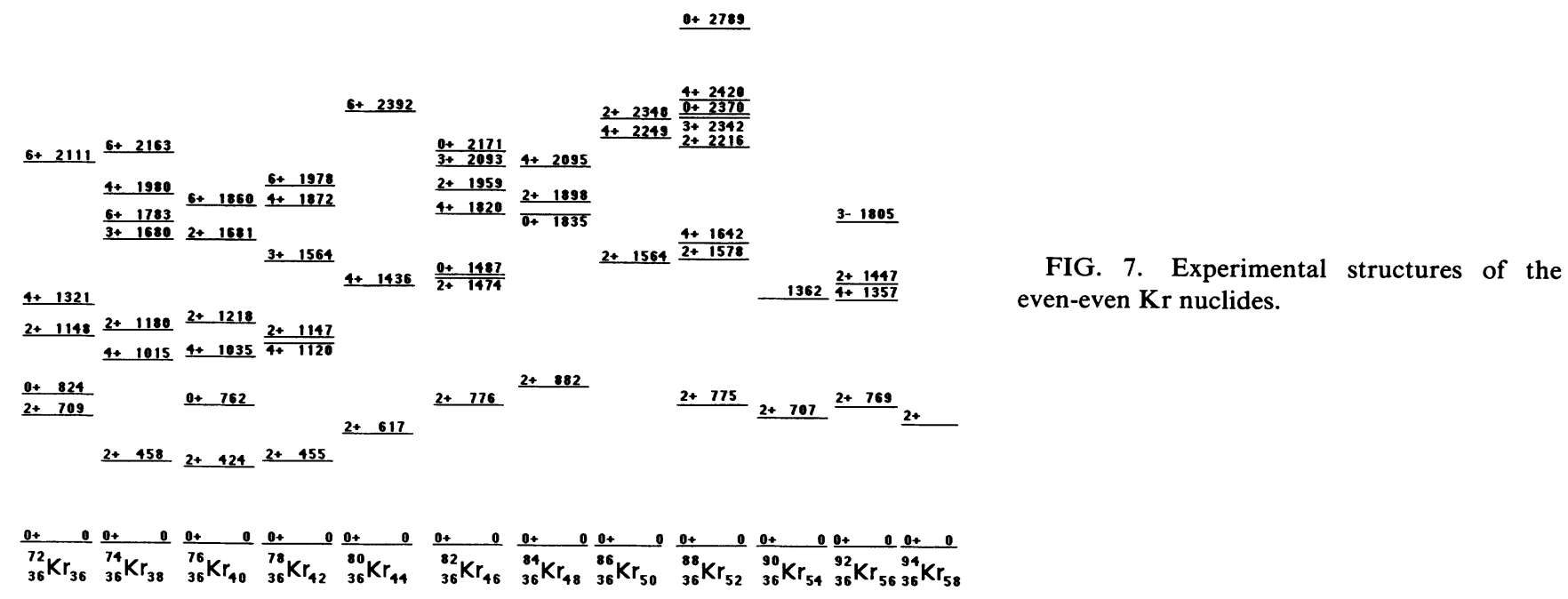


TABLE III. Deformation parameters $\varepsilon_{2}$. For each mass number, the first line shows prolate deformation $(P)$ and the second line oblate deformation $(O)$. For TRS $\varepsilon_{2}$ has been recalculated from $\beta_{2}$ in the original calculation.

\begin{tabular}{|c|c|c|c|c|c|c|}
\hline$A$ & TRS $(\mathrm{Kr})$ & TRS (Se) & PTR-MO & PTR-WS & VMI(S) & $\operatorname{VMI}(A)$ \\
\hline $81 P$ & & & 0.20 & 0.18 & 0.21 & 0.22 \\
\hline $79 P$ & & & 0.22 & 0.20 & 0.24 & 0.24 \\
\hline $78 P$ & 0.28 & & & & & \\
\hline$O$ & 0.21 & & & & & \\
\hline $77 P$ & & & 0.30 & & 0.29 & 0.27 \\
\hline $76 P$ & 0.31 & & & & & \\
\hline$O$ & 0.23 & & & & & \\
\hline $75 P$ & & & 0.325 & & 0.32 & 0.32 \\
\hline $74 P$ & 0.32 & 0.27 & & & & \\
\hline$O$ & 0.27 & 0.21 & & & & \\
\hline $73 P$ & & 0.34 & & & 0.32 & 0.32 \\
\hline$O$ & & 0.215 & & & & \\
\hline $72 P$ & 0.31 & 0.29 & & & & \\
\hline$O$ & 0.28 & 0.23 & & & & \\
\hline $71 P$ & & & 0.31 & & 0.31 & \\
\hline$O$ & & & 0.20 & & & \\
\hline $70 P$ & & 0.23 & & & & \\
\hline$O$ & & 0.24 & & & & \\
\hline
\end{tabular}

rearrangement of the $3 p_{1 / 2}$ and $3 f_{5 / 2}$ subshells which then prevents the shift of the Fermi level between the [301] $\frac{3}{2}$ and [312] $\frac{3}{2}$ orbitals, which is crucial to the interpretation of the ground-state magnetic moments of ${ }^{75-81} \mathrm{Br}$. The band arising from the [440] $\frac{1}{2}$ orbital is well known to have a $\frac{5}{2}^{+}$bandhead and so cannot produce a spin- $\frac{1}{2}$ ground state.

In Fig. 9 we show the results of calculations, using the PTR-MO with constant moment of inertia, of the lowest energy levels with $I^{\pi}=\frac{1}{2}^{-}, \frac{3}{2}^{-}, \frac{5}{2}^{-}$in ${ }^{71,73} \mathrm{Br}$, as a function of $\gamma$, for three representative values of $\varepsilon_{2}$. The calculation was performed for a large number of deformation values covering the region $0.20 \leq \varepsilon_{2} \leq 0.34$. The results show that, for ${ }^{73} \mathrm{Br}$, the $\frac{1}{2}^{-}$ground state arises in the whole region of deformation, provided $\gamma \geq 30^{\circ}$. With decreasing $\varepsilon_{2}, \gamma$ approaches $60^{\circ}$. In ${ }^{71} \mathrm{Br}$, the $\frac{5}{2}^{-}$ground state is first calculated for $\varepsilon_{2}=0.21$ and $\gamma>30^{\circ}$. Howev- er, recent experimental data $[21,22]$ provided another criterion to be satisfied, namely close proximity of a $\frac{5}{2}^{-}$ state $(27 \mathrm{keV})$ to the $\frac{1}{2}^{-}$ground state in ${ }^{73} \mathrm{Br}$ and of a $\frac{1}{2}^{-}$ state $(9 \mathrm{keV})$ to the $\frac{5}{2}^{-}$ground state in ${ }^{71} \mathrm{Br}$. Figure 6 shows that this level structure can be achieved in this deformation region for both nuclei only for $\gamma$ close to $60^{\circ}$ and that the two lowest-energy states in ${ }^{71,73} \mathrm{Br}$ can be best understood as pure oblate states. The structure of these states is very similar in both nuclides. The $\frac{1}{2}^{-}$state has two major components, $75 \%(83 \%)$ [310] $\frac{1}{2}$ and $23 \%$ (15\%) [321] $\frac{1}{2}$ in ${ }^{71} \mathrm{Br}\left({ }^{73} \mathrm{Br}\right)$. The makeup of the $\frac{5}{2}^{-}$state is more complicated. It results from strong Coriolis interaction among orbitals of $2 f_{5 / 2}$ and $3 p_{3 / 2}$ spherical origin. The main component is $43 \%(45 \%)$ [ 312$] \frac{5}{2}$ with contributions from $\frac{5}{2}^{-}$members of bands built on [321] $\frac{3}{2}$, $[321] \frac{1}{2}$, and $[310] \frac{1}{2}$ states $26 \%(24 \%), 18 \%(16 \%)$, and $10 \%(14 \%)$, in ${ }^{71} \mathrm{Br}\left({ }^{73} \mathrm{Br}\right)$. The calculated magnetic mo-

TABLE IV. Experimental and calculated magnetic dipole moments, electric quadrupole moments, and values of $B\left(E 2 ; \frac{7}{2}^{-} \rightarrow \frac{3}{2}^{-}\right)$and $B\left(E 2 ; \frac{13}{2}^{-} \rightarrow \frac{9}{2}^{-}\right)$for the $\frac{3}{2}^{-}$band in ${ }^{73-81} \mathrm{Br}$. Experimental data obtained from the current Nuclear Data Sheets and the present work. (a) $B\left(E 2 ; \frac{7}{2}^{-} \rightarrow \frac{3}{2}^{-}\right)$(W.u.); (b) $B\left(E 2 ; \frac{13}{2}^{-} \rightarrow \frac{9}{2}^{-}\right)$(W.u.).

\begin{tabular}{|c|c|c|c|c|c|c|}
\hline$A$ & $\exp$ & PTR-MO & PTR-WS (Kr) & PTR-WS (Se) & VMI(S) & VMI(A) \\
\hline $81 \mu\left(\mu_{N}\right)$ & +2.27 & 2.28 & $2.13^{\mathrm{a}}$ & & 2.25 & 2.28 \\
\hline$Q(e \mathrm{~b})$ & $+0.276(4)$ & 0.26 & $0.27^{\mathrm{a}}$ & & 0.28 & 0.28 \\
\hline $79 \mu\left(\mu_{N}\right)$ & +2.11 & 2.14 & $2.01^{\mathrm{a}}$ & & 2.16 & 2.11 \\
\hline$Q(e \mathrm{~b})$ & $+0.331(4)$ & 0.33 & $0.29^{\mathrm{a}}$ & & 0.34 & 0.33 \\
\hline $77 \mu\left(\mu_{N}\right)$ & $+0.92(5)$ & 0.93 & 0.70 & & 1.02 & 0.93 \\
\hline (a) & $35(9)$ & 52 & 40 & & 47 & 33 \\
\hline (b) & $68(5)$ & 106 & 80 & & 99 & 81 \\
\hline $75 \mu\left(\mu_{N}\right)$ & $+0.76(18)$ & 0.86 & 0.68 & 0.77 & 0.89 & 0.75 \\
\hline (a) & $61(5)$ & 64 & 53 & 37 & 61 & 60 \\
\hline (b) & $148(+66,-34)$ & 128 & 104 & 73 & 125 & 120 \\
\hline 73 (a) & $45(6)$ & 72 & 57 & 42 & 60 & 60 \\
\hline (b) & $168(+28,-21)$ & 143 & 111 & 86 & 121 & 121 \\
\hline
\end{tabular}

${ }^{\text {a }}$ Deformation not available from TRS calculations (see text). 
TABLE V. Structure of the $\frac{3}{2}^{-}$bandhead in ${ }^{71-81} \mathrm{Br}$. Components of the total wave function are given in percent. (i) [312] $\frac{3}{2}$; (ii) [301] $\frac{3}{2}$.

\begin{tabular}{rrrrr}
\hline \hline \multicolumn{1}{c}{$\boldsymbol{A}$} & PTR-MO & PTR-WS $($ Kr) & VMI(S) & VMI(A) \\
\hline 81 (i) & 25 & 6 & 18 & 8 \\
(ii) & 67 & 88 & 74 & 84 \\
79 (i) & 46 & 20 & 47 & 33 \\
(ii) & 50 & 74 & 46 & 58 \\
77 (i) & 98 & 86 & 98 & 95 \\
(ii) & $<1$ & 1 & $<1$ & 4 \\
75 (i) & 98 & 92 & 98 & 98 \\
(ii) & $<1$ & $<1$ & $<1$ & 1 \\
73 (i) & 98 & 98 & 98 & 98 \\
(ii) & $<1$ & $<1$ & $<1$ & 1 \\
71 (i) & 98 & 98 & 98 & \\
(ii) & $<1$ & $<1$ & $<1$ & \\
\hline \hline
\end{tabular}

ment of the $\frac{5}{2}^{-}$ground state in ${ }^{71} \mathrm{Br}$ for the proposed oblate shape is about $1.5 \mu_{N}$.

Examination of Table III shows that the deformation parameters for prolate shapes fitted using PTR-MO (with both the constant and variable moment of inertia) agree very well with the corresponding TRS values for $\mathrm{Kr}$ cores, but show poorer agreement for Se cores. This result suggests that the actual core of the odd- $A \mathrm{Br}$ (for prolate shapes) is quite close to the shape of the neighboring even-even $\mathrm{Kr}$ nucleus and there is very little core polarization. Deformations found for oblate shapes in ${ }^{71,73} \mathrm{Br}$ are very similar to those calculated for oblate $\mathrm{Se}$ cores and are about $20 \%$ smaller than those for an oblate $\mathrm{Kr}$ core. The TRS calculations do not predict any deviation from axial symmetry in even-even Se and $\mathrm{Kr}$, which is consistent with our findings that the negative parity states in odd- $A \mathrm{Br}$ can be described without a need for $\gamma$ different from $0^{\circ}$ or $60^{\circ}$. In summary, our finding of large prolate deformation of lighter odd- $A \mathrm{Br}$ isotopes starting to develop at ${ }^{77} \mathrm{Br}$ agrees very well with predictions of TRS calculations. Our fits suggest that the deformation of the oblate coexisting states in ${ }^{71,73} \mathrm{Br}$ is considerably smaller than that of the prolate states. Differences in extracted deformations for each odd- $A$ nuclide in question are very small and reflect the effect of different models. It is quite remarkable how self-consistent are the results of all models used. The choice of moment of inertia is not expected to influence ground-state properties in the first approximation, but even the alternative choice of the single-particle parameters $k, \mu$ in the particle-rotor calculation makes very little difference in predictions of electromagnetic properties.

As a result of our analysis, a coherent description for the low-energy levels in ${ }^{71-81} \mathrm{Br}$ can be drawn. Going from ${ }^{81} \mathrm{Br}$ to ${ }^{75} \mathrm{Br}$, we find a smooth change in prolate ground-state structure from [301] $\frac{3}{2}$ to almost pure [312] $\frac{3}{2}$ configuration. The structure in ${ }^{71,73} \mathrm{Br}$ can be understood in terms of shape coexistence between a less deformed oblate ground state and a more deformed prolate $\frac{3}{2}^{-}$yrast band. In both the neighboring Se and $\mathrm{Kr}$ even-even nuclides, TRS calculations predict similar coexistence. It follows that there is a prolate to oblate ground-state shape transition between ${ }^{75} \mathrm{Br}$ and ${ }^{73} \mathrm{Br}$.

\section{B. The odd-odd Br nuclides}

\section{Structure of ${ }^{76} \mathrm{Br}$}

We now turn to the odd-odd bromine nuclei. The static electromagnetic moments of the $1^{-}$ground state of ${ }^{76} \mathrm{Br}$ have been previously measured to be $\mu= \pm 0.5482(1) \mu_{N}$ and $Q= \pm 0.27(1)$ b [14]. The quadrupole moment implies a deformation of $\varepsilon=0.31, \gamma=0$

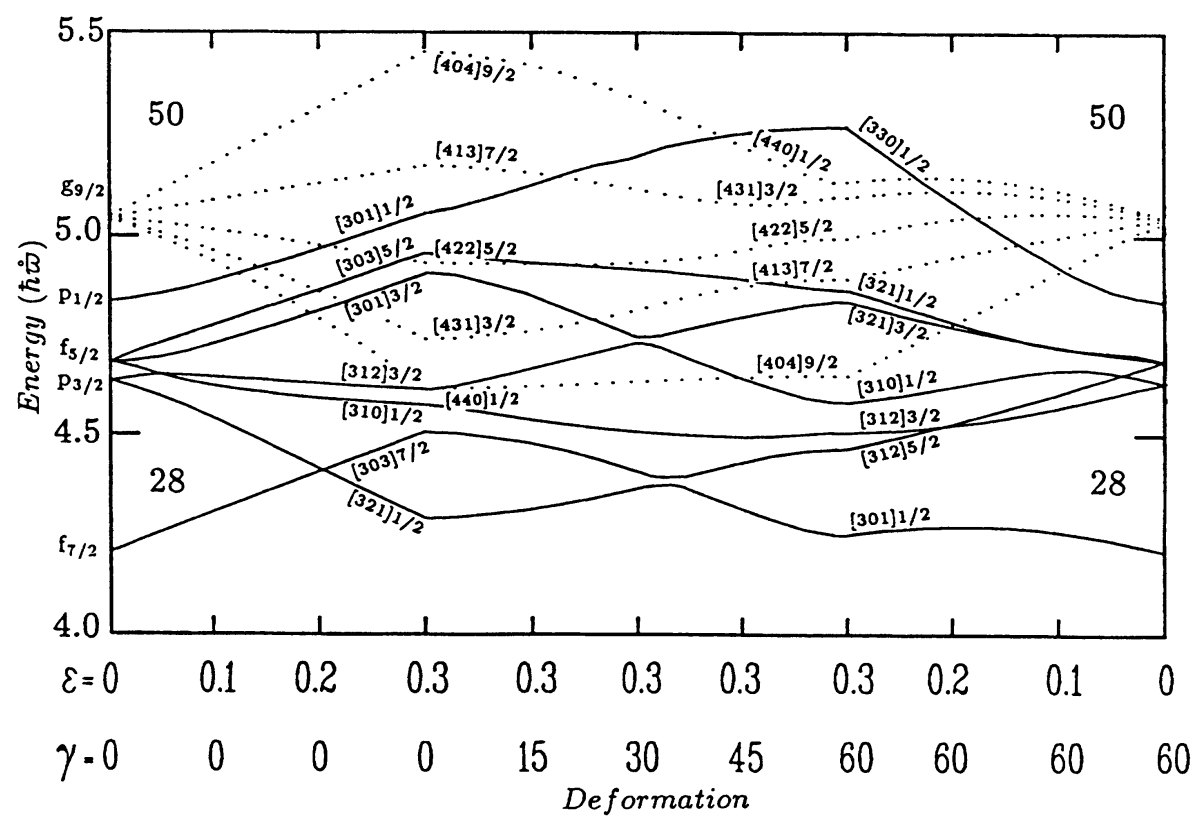

FIG. 8. Proton single-particle levels in the $A \sim 75$ mass region in the $(\varepsilon, \gamma)$ plane. The Nilsson modified oscillator parameters $\kappa=0.068$ and $\mu=0.38$ are used. 


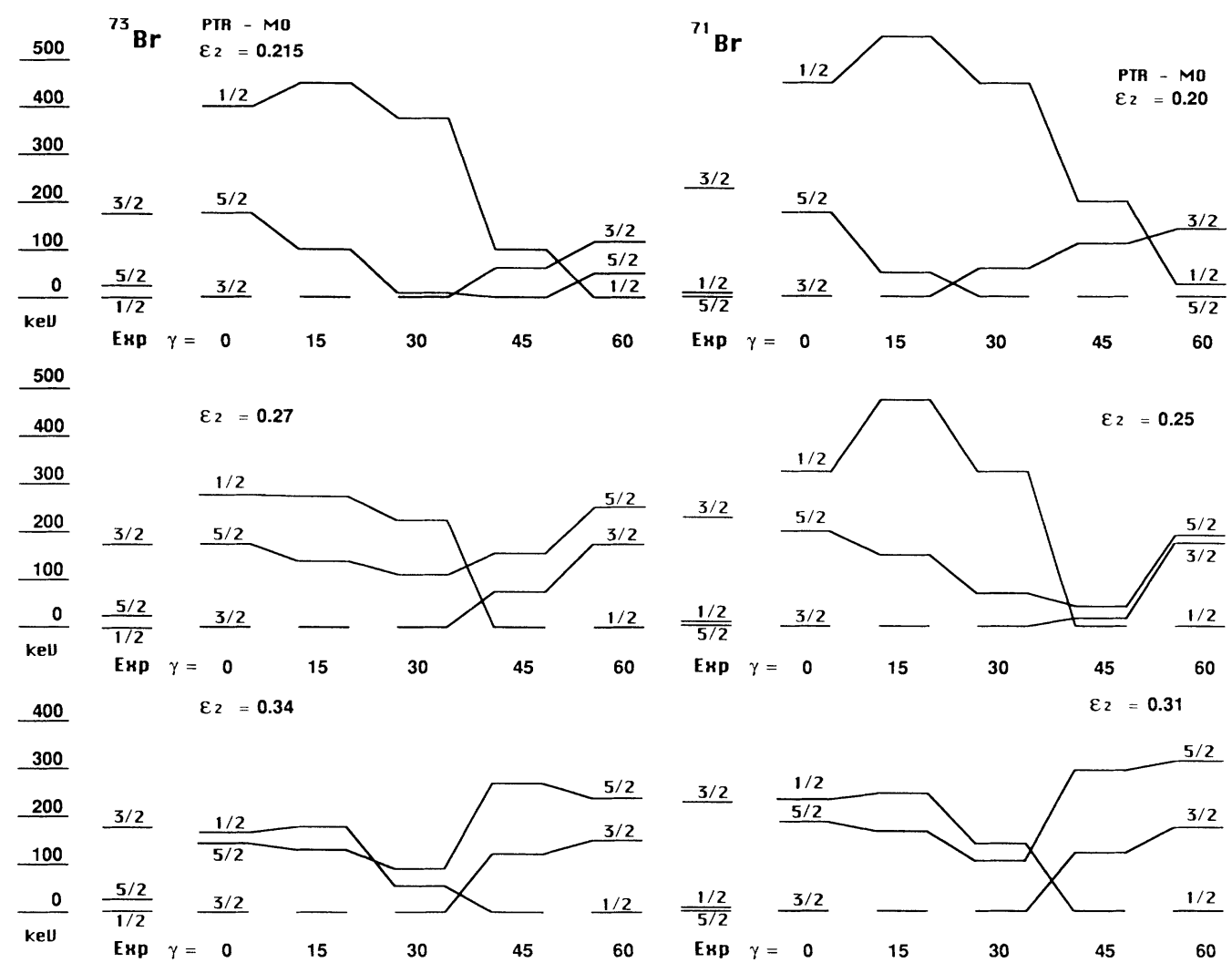

FIG. 9. The relative positions of the lowest $\frac{1}{2}^{-}, \frac{3}{2}^{-}$, and $\frac{5}{2}^{-}$bandheads in ${ }^{71,73} \mathrm{Br}$ calculated with the PTR-MO model with constant moment of inertia for three different values of $\varepsilon_{2}$ as a function of $\gamma$.

which fits in well with the systematics of the odd- $A$ nuclear deformations given in Table IV. With this deformation, the particle-rotor model indeed leads to a $1^{-} \pi$ $[312] \frac{3}{2} v[422] \frac{5}{2} K=1$ ground-state configuration, with predicted moments of $-0.77 \mu_{N}$ and $Q=+0.27 \mathrm{~b}$. These values are consistent with the previous configuration assignment of Ekstrom et al. [17]. (Note that the $\pi[301] \frac{3}{2} v[422] \frac{5}{2} K=1$ configuration is associated with a much larger magnetic moment of $-1.5 \mu_{N}$ and, according to the Gallagher-Moszkowski selection rule, could not give rise to a $1^{-}$ground state [34].)

\section{Structure of ${ }^{74} \mathrm{Br}$}

As was mentioned previously, the parity assignment to the spin-4 isomeric state in ${ }^{74} \mathrm{Br}$ is not fully established. Negative parity has been proposed on the basis of supposed first unique forbidden $\beta^{+} / \mathrm{EC}$ transitions to ${ }^{74} \mathrm{Se}$ [35] while positive parity has been suggested from a comparison with the $4^{+}$and $4^{-}$band structures in ${ }^{76} \mathrm{Br}$ $[36,37]$. In view of the fact that the corresponding neutron Fermi levels are virtually coincident in the region $0.3<\varepsilon<0.4$, as can be seen from Fig. 4, such similarities between the states of ${ }^{74,76} \mathrm{Br}$ might well be expected.

Considering first the case of negative parity, likely candidates for a $4^{-}$isomeric state include the $\pi[312] \frac{3}{2} v[422] \frac{5}{2}, \pi[440] \frac{1}{2} v[301] \frac{3}{2}$, and $\pi[440] \frac{1}{2} v[303] \frac{5}{2}$ orbitals. However, the calculated moments of these configurations $-0.16 \mu_{N},+3.93 \mu_{N}$, and $+4.20 \mu_{N}$, re- spectively, are all incompatible with the experimental moment of $\pm 1.82(1) \mu_{N}$. The tentatively proposed $\pi[301] \frac{3}{2} v[422] \frac{5}{2}$ orbital [17], with a theoretical moment of $+1.71 \mu_{N}$, never comes close enough to the Fermi level to be a viable possibility.

By contrast, the moment of the positive parity $\pi[431] \frac{3}{2} v[422] \frac{5}{2}$ configuration is calculated to be $+1.77 \mu_{N}$, in excellent agreement with the experimental value of $1.82(1) \mu_{N}$. Therefore the value of the measured magnetic moment strongly favors a $4^{+}$assignment for the isomeric state. The positive parity assignment and the indication that the orbitals are both of positive parity are also favored by Holcomb et al. [37], on the basis of the fast $E 2$ transitions that they observe, as well as on the similarity of the overall behavior of the band that is built on this isomer to those better defined bands in ${ }^{76} \mathrm{Br}$ $[38,39]$.

\section{Structure of ${ }^{72} \mathrm{Br}$}

Recent in-beam gamma spectroscopy studies on ${ }^{72} \mathrm{Br}$ have revealed three rotational bands [40]. Electric transition strengths within the band terminating on the $1^{-}$ isomeric state indicate a nuclear deformation in the region $|\varepsilon| \sim 0.30$. Shape coexistence at low spin is a real possibility, and interestingly, no band structure clearly associated with the $3^{+}$ground state has been observed, reminiscent of the neighboring nucleus ${ }^{73} \mathrm{Br}$. With this structure in mind, a search for a possible $3^{+}$ground state 
was carried out for a mesh of prolate, oblate, and triaxial deformation points in the range $0.05 \leq \varepsilon \leq 0.40, \gamma=0^{\circ}$, $15^{\circ}, 25^{\circ}, 35^{\circ}, 45^{\circ}$, and $60^{\circ}$. Despite this extensive search no such ground state was found, nor did any low energy $3^{+}$states have moments consistent with the small measured value of $\pm 0.55(21) \mu_{N}$. A similar search for an isomeric $1^{-}$state at large deformation also failed to produce an obvious candidate. At lower deformation, however, the above-mentioned $\pi[301] \frac{3}{2} v[422] \frac{5}{2}$ orbital [17] with a calculated moment of $+1.71 \mu_{N}$ is a possibility, but it is not favored by the Gallagher-Moszkowski rules.

On the other hand, examination of the structures of the adjacent odd-mass nuclides ${ }^{71} \mathrm{Br}$ and ${ }^{71} \mathrm{Se}[41]$ indicates a much less deformed shape for the possible $3^{+}$ground state of ${ }^{72} \mathrm{Br}$. As noted above, the observed structure of ${ }^{71} \mathrm{Br}$ can be described by a nearly pure oblate structure calculation. Likewise, the calculations of Zhao support an oblate structure for the levels of ${ }^{71} \mathrm{Se}[42]$. Alternately, these states could be even less deformed and have shell model $f_{5 / 2}$ and $p_{1 / 2}$ configurations. The latter singleparticle structure would offer some explanation as to why the deformed particle-rotor model could not provide an adequate description for the structure of ${ }^{72} \mathrm{Br}$.

At small deformation where the multiplet splitting for odd-odd nuclides can be described by the long range quadrupole interaction and longer range spin-dipole interaction as suggested by Paar [43], there would be a low-energy $\pi f_{5 / 2} v f_{5 / 2}$ multiplet with spins ranging from $0^{+}$to $5^{+}$and two $2^{+}, 3^{+}$doublets, the $\pi f_{5 / 2} v p_{1 / 2}$ and the other $\pi p_{1 / 2} v f_{5 / 2}$. For both of these doublets, the low-energy state would be the $3^{+}$level. Empirical moments for these configurations are $2.14 \mu_{N}$ and $0.74 \mu_{N}$, respectively, using a $\pi f_{5 / 2}$ moment of $1.6 \mu_{N}$ from the study of moments in odd-mass As nuclides [44-46]. Even using $\pi f_{5 / 2}$ the Schmidt limit value of $0.84 \mu_{N}$ only brings the calculated $\pi f_{5 / 2} v p_{1 / 2}$ moment down to $1.42 \mu_{N}$. The measured moment of $0.55(21) \mu_{N}$ is decisive between these two configurations in favor of the $\pi p_{1 / 2} v f_{5 / 2}$ configuration. The empirical moment for the $3^{+}$member of the $\pi f_{5 / 2} v f_{5 / 2}$ multiplet is $1.49 \mu_{N}$, indicating little contribution from that configuration. For the $1^{-}$level, it can be seen that various combinations involving the $\frac{5}{2}^{+}$level at $670 \mathrm{keV}$ in ${ }^{71} \mathrm{Br}$ and $\frac{3}{2}^{-}$levels in

${ }^{71} \mathrm{Se}$ would produce a $1^{-}$level and that level would show a larger moment owing to the involvement of the positive parity $\frac{5}{2}^{+}$level, whatever its origin. It is not at all clear why this possibility would be isomeric, however, and why it should lie at a lower energy than the $2^{+}$member of the $f p$ doublets. It seems much more likely that the $1^{-}$band is considerably more deformed than the $3^{+}$band and not well described by the Paar model.

\section{CONCLUSION}

In the odd- $\boldsymbol{A}$ nuclei it has been shown that there is a transformation in the character of the ground-state configuration from the less deformed [301] $\frac{3}{2}$ orbital in ${ }^{79,81} \mathrm{Br}$ to the more deformed [312] $\frac{3}{2}$ orbital in ${ }^{75,77} \mathrm{Br}$. The measured magnetic dipole moments are well reproduced by the theory and are fully consistent with the de- formations suggested by the electric quadrupole data. While these magnetic moments are only compatible with prolate deformations, the ground-state spin of ${ }^{73} \mathrm{Br}$ can only be interpreted in terms of an oblate nuclear shape. This fact provides strong evidence in the bromine nuclei for the conjectured prolate-oblate shape transition [1]. (In fact the transition was predicted to occur as high as ${ }^{78} \mathrm{Br}$ which we have shown not to be the case.) The presence in ${ }^{73} \mathrm{Br}$ of a low-energy $\frac{3}{2}^{-}$rotational band, whose nature is similar to that of the ground-state band in ${ }^{75} \mathrm{Br}$, indicates the occurrence of coexisting oblate and prolate shapes.

In the odd-odd nuclei, the moment of the ${ }^{76} \mathrm{Br}$ ground state demonstrates the involvement of a $\pi[312] \frac{3}{2}$ orbital as observed in the neighboring odd- $A$ nuclei, ${ }^{75,77} \mathrm{Br}$. The magnetic moment of ${ }^{74} \mathrm{Br}^{m}$ also suggests a positive parity assignment for the spin-4 isomeric state and the transition rates in the band favor a combination of positive parity proton and neutron orbitals from the $g_{9 / 2}$ singleparticle orbitals as opposed to combinations of the negative parity orbitals. The structure and moments for ${ }^{72} \mathrm{Br}$ suggest that the maximum deformation in $\mathrm{Br}$ lies in the region of ${ }^{74,75,76} \mathrm{Br}_{39,40,41}$ and that the lower mass $\mathrm{Br}$ nuclides revert to oblate and more spherical shape for $N \leq 38$.

The spectacular change in the moments for the $\frac{3}{2}^{-}$ ground states of the ${ }_{35} \mathrm{Br}$ nuclides, together with the large quantity of in-beam and decay data, aid in attributing nuclear deformation to the occupancy of the low- $K$ downsloping positive parity orbitals from the $g_{9 / 2}$ proton and neutron orbitals. For those structures where there is little occupancy of either the proton or neutron $g_{9 / 2}$ orbitals as found for ${ }^{68,70,71} \mathrm{Se}_{34,36,37}$ deformation at low energy appears to be small and largely oblate in character. The odd- $Z{ }_{33}$ As nuclides are an example of the fact that the moments for the $f_{5 / 2}$ levels increase as $N$ approaches 40 where there is $g_{9 / 2}$ neutron occupancy to induce $g_{9 / 2}$ proton occupancy. In both the light and heavy As nuclides, where the $g_{9 / 2}$ neutron orbitals are either full or empty, the moments move back to lower values nearer the Schmidt limits.

Thus, it is possible to observe that the $\mathrm{Br}$ nuclides lie exactly in the transition region between weakly deformed structures observed in the As and Se nuclides and the more deformed $\mathrm{Kr}$ and $\mathrm{Rb}$ nuclides. Our new particlerotor calculations show that the addition of the 35 th proton tips the balance at midshell toward deformation and that the $N=39,40,41 \mathrm{Br}$ nuclides can be best described as a hole in the more deformed $\mathrm{Kr}$ nuclides rather than a particle coupled to the less deformed Se nuclides.

\section{ACKNOWLEDGMENTS}

The authors wish to express their gratitude to Dr. K. Schlösser and the members of the ISOLDE Collaboration for their efforts in the production of the ${ }^{76,77} \mathrm{Br}$ samples and to NATO for travel support. J.R. wishes to thank the U.K. SERC for financial support, and W.W. is grateful for support from the John Simon Guggenheim Memorial Foundation. 
[1] R. Bengtsson, P. Möller, J. R. Nix, and Jing-ye Zhang, Phys. Scr. 29, 402 (1984).

[2] W. Nazarewicz, J. Dudek, R. Bengtsson, and I. Ragnarsson, Nucl. Phys. A435, 397 (1985).

[3] L. Luhmann, M. Debray, K. P. Lieb, W. Nazarewicz, and B. Wörmann, Phys. Rev. C 31, 828 (1985).

[4] K. P. Lieb and J. J. Kolata, Phys. Rev. C 15, 939 (1977).

[5] R. B. Piercey, A. V. Ramayya, R. M. Ronningen, J. H. Hamilton, V. Maruhn-Rezwani, R. L. Robinson, and H. J. Kim, Phys. Rev. C 19, 344 (1979).

[6] N. J. Stone, C. J. Ashworth, I. S. Grant, A. G. Griffiths, S. Ohya, J. Rikovska, and P. M. Walker, in Proceedings of the Conference on Nuclear Structure of the Zirconium Region, Bad Honnef, 1988, edited by J. Eberth, R. A. Meyer, and K. Sistemich (Springer-Verlag, Berlin, 1988), p. 309.

[7] A. Griffiths, C. J. Ashworth, I. S. Grant, J. Rikovska, N. J. Stone, P. M. Walker, W. B. Walters, and J. P. White, Hyperfine Interact. 43, 481 (1988).

[8] D. I. Bradley, J. P. White, C. J. Ashworth, N. J. Stone, and J. Rikovska, Hyperfine Interact. 35, 1033 (1987).

[9] N. J. Stone and H. Postma, in Low Temperature Nuclear Orientation, edited by N. J. Stone and H. Postma (NorthHolland, Amsterdam, 1987), pp. 3-30.

[10] P. Herzog, U. Dämmrich, K. Freitag, C. D. Herrmann, and K. Schlösser, Z. Phys. B 64, 353 (1986).

[11] P. Herzog, U. Dämmrich, K. Freitag, B. Prillwitz, J. Prinz, and K. Schlösser, Nucl. Instrum. Methods B26, 471 (1987).

[12] R. B. Alexander, P. T. Callaghan, and J. M. Poate, Phys. Rev. B 9, 3022 (1974).

[13] P. T. Callaghan, N. J. Stone, and R. B. Alexander, Hyperfine Interact. 3, 267 (1977).

[14] C. M. Lederer and V. S. Shirley, Table Of Isotopes, 7th ed. (Wiley, New York, 1978).

[15] J. Prinz, I. Berkes, P. Herzog, B. Hilmi, M. de Jesus, M. Massaq, I. Romanski, and the NICOLE and ISOLDE Collaborations, Hyperfine Interact. (in press).

[16] L. P. Ekstrom, Nucl. Data Sheets 32, 211 (1981).

[17] C. Ekstrom and L. Robertsson, Phys. Scr. 22, 344 (1980).

[18] M. M. King, Nucl. Data Sheets 51, 161 (1987).

[19] K. Heiguchi, S. Mitarai, B. J. Min, and T. Kuroyanagi, Nucl. Phys. A474, 484 (1987).

[20] J. Heese, K. P. Lieb, L. Lühmann, S. Ulbig, B. Wörmann, D. Alber, H. Grawe, H. Haas, and B. Spellmeyer, Phys. Rev. C 36, 2409 (1987).

[21] J. Heese, N. Martin, C. J. Gross, W. Fieber, K. P. Lieb, A. Kuhnert, K. H. Maier, and X. Sun, Phys. Rev. C 41, 1553 (1990).

[22] J. W. Arrison, T. Chapuran, U. J. Hüttmeier, and D. P. Balamuth, Phys. Lett. B 248, 39 (1990).

[23] G. Garcia-Bermudez, C. Baktash, A. J. Kreiner, and M. A. J. Mariscotti, Phys. Rev. C 25, 1396 (1982).

[24] B. Singleton, private communication.
[25] T. Mylaeus, J. Busch, J. Egerth, M. Liebchen, R. Sefzig, S. Skoda, W. Teichert, M. Wiosna, P. von Brentano, K. Schiffer, K. O. Zell, A. V. Ramayya, K. H. Maier, H. Grawe, A. Kluge, and W. Nazarewicz, J. Phys. G 15, L135 (1989).

[26] N. Nadon, A. D. Jaafri, J. De Buc, B. Ferraoun, G. Kajrys, P. Lariviere, J. Mailloux, D. Prevost, S. Pilotte, A. Tehami, S. Monaro, and J. C. Waddington, private communication.

[27] J. W. Arrison, Ph.D. thesis, University of Pennsylvania, 1990 (unpublished).

[28] S. E. Larsson, G. Leander, and I. Ragnarsson, Nucl. Phys. A307, 189 (1978).

[29] A. G. Griffiths, Ph.D. thesis, Oxford University, 1988 (unpublished).

[30] M. A. J. Mariscotti, G. Scharff-Goldhaber, and B. Buck, Phys. Rev. 178, 1864 (1969).

[31] T. Bengtsson and I. Ragnarsson, Nucl. Phys. A439, 14 (1985).

[32] R. Wyss, private communication.

[33] R. Wyss, W. Satula, W. Nazarewicz, and A. Johnson, Nucl. Phys. A511, 324 (1990).

[34] C. J. Gallagher and S. A. Moszkowski, Phys. Rev. 111, $1282(1958)$

[35] A. Coban, J. C. Lisle, G. Murray, and J. C. Willmott, Part. Nucl. 4, 108 (1972).

[36] G. Winter, J. Döring, W. D. Fromm, L. Funke, P. Kemnitz, and E. Will, Z. Phys. A 309, 243 (1983).

[37] J. W. Holcomb, T. D. Johnson, P. C. Womble, P. D. Cottle, S. L. Tabor, F. E. Durham, and S. G. Buccino, Phys. Rev. C 43, 470 (1991).

[38] D. F. Winchell, J. X. Saldin, M. S. Kaplan, and H. Takai, Phys. Rev. C 41, 1264 (1990).

[39] S. G. Buccino, F. E. Curham, J. W. Holcomb, T. D. Johnson, P. D. Cottle, and S. L. Tabor, Phys. Rev. C 41, 2056 (1990).

[40] S. Ulbig, F. Cristancho, J. Heese, K. P. Lieb, T. Osipowicz, B. Wörmann, J. Eberth, T. Mylaeus, and $\mathbf{M}$. Wiosna, Z. Phys. A 329, 51 (1988).

[41] A. V. Ramayya, Nucl. Instrum. Methods B40/41, 432 (1989).

[42] X. Zhao, Ph.D. thesis, Vanderbilt University, 1989 (unpublished).

[43] V. Paar, Nucl. Phys. A331, 16 (1979).

[44] P. Herzog, N. J. Stone, and P. D. Johnson, Nucl. Phys. A259, 378 (1976).

[45] N. Severijns, E. van Walle, D. Vandeplassche, J. Wouters, J. Vanhaverbeke, W. Vanderpoorten, and L. Vanneste, in Proceedings of the Conference on Nuclear Structure of the Zirconium Region [6], p. 245.

[46] W. Hogervorst, H. A. Helms, G. J. Zaal, J. Bouma, and J. Blok, Z. Phys. A 294, 1 (1980). 